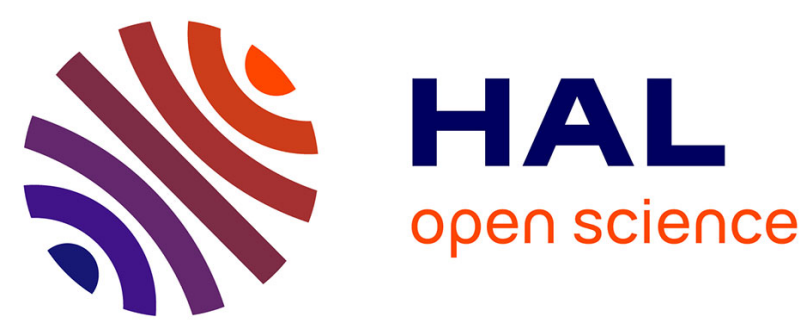

\title{
Parametrized hyperplane arrangements for control design with collision avoidance constraints
}

Daniel Ioan, Sorin Olaru, Ionela Prodan, Florin Stoican, Silviu-Iulian Niculescu

\section{- To cite this version:}

Daniel Ioan, Sorin Olaru, Ionela Prodan, Florin Stoican, Silviu-Iulian Niculescu. Parametrized hyperplane arrangements for control design with collision avoidance constraints. 15th IEEE International Conference on Control and Automation (ICCA), Jul 2019, edinburgh, United Kingdom. 10.1109/ICCA.2019.8899977 . hal-02329329

\section{HAL Id: hal-02329329}

https://hal-centralesupelec.archives-ouvertes.fr/hal-02329329

Submitted on 10 Apr 2020

HAL is a multi-disciplinary open access archive for the deposit and dissemination of scientific research documents, whether they are published or not. The documents may come from teaching and research institutions in France or abroad, or from public or private research centers.
L'archive ouverte pluridisciplinaire HAL, est destinée au dépôt et à la diffusion de documents scientifiques de niveau recherche, publiés ou non, émanant des établissements d'enseignement et de recherche français ou étrangers, des laboratoires publics ou privés. 


\title{
Parametrized Hyperplane Arrangements for Control Design with Collision Avoidance Constraints
}

\author{
Daniel Ioan ${ }^{1}$, Sorin Olaru ${ }^{1}$, Ionela Prodan ${ }^{2}$, Florin Stoican ${ }^{3}$, Silviu-Iulian Niculescu ${ }^{1}$
}

\begin{abstract}
This paper concerns the problem of collision avoidance in a dynamical multi-obstacle environment. The design problem is commonly stated in the literature in terms of a receding-horizon constrained optimization problem over a non-convex domain. Preliminary results based on hyperplane arrangements lead to a mixed-integer formulation of the problem. This formalism is adequate for a static multiobstacle environment, but may be impractical in a dynamical context. Nevertheless, this shortcoming can be alleviated by considering an additional analysis step and by an appropriate proper choice of the representation of the environment in a time-varying framework. The present paper tackles this issue by using zonotopes and discussing their parametrization in a collision avoidance type of application.
\end{abstract}

\section{INTRODUCTION}

Collision-free control of autonomous agents within a multi-obstacle environment is at the forefront of research interests in the control community [1] and takes multiple forms according to the surveillance, rescue or monitoring type of application. Moreover, the navigation through dynamical environments is a challenging task because of the complexity issues, the intrinsic quality of the information shared within the system or the practical controlability or physical limitations to be dealt with.

The classical modeling approaches (graph- or samplebased [2]), usually characterized by heuristic aspect, have been included in the control strategies proposed for the resolution of the collision avoidance problem. MPC (Model Predictive Control) is an often used strategy in this domain. Being an optimization-based control strategy, MPC has the capability to cope either directly [3], [4] or indirectly [5], [6] with the inherently non-convex collision avoidance constraints.

In what follows, we concentrate on a direct method, formulating the MPC problem as a receding horizon Mixedinteger Program (MIP). More precisely, we consider convex polyhedral sets in order to model the obstacles and to characterize the resulting non-convex feasible domain as a union of cells, via hyperplane arrangements [7]. Nevertheless, in many practical applications, as autonomous overtaking [8] or coordination of vehicles at intersections [9], we have to deal

\footnotetext{
${ }^{1}$ Laboratory of Signals and Systems, Univ. Paris-Sud-CentraleSupelecCNRS, Université Paris Saclay. \{daniel.ioan, sorin.olaru, silviu.niculescu\}@l2s.centralesupelec.fr

${ }^{2}$ Univ. Grenoble Alpes, Grenoble INP^, LCIS, F-26000, Valence, France. ionela.prodan@lcis.grenoble-inp. fr

* Institute of Engineering Univ. Grenoble Alpes.

${ }^{3}$ Department of Automatic Control and Systems Engineering, UPB, Romania florin.stoicandacse.pub.ro
}

with dynamical obstacles. Therefore, the resulting hyperplane arrangement is time-varying. Since a re-computation of the arrangement at each time instant may be impractical in terms of complexity, the question to be addressed is: How can one use hyperplane arrangements for the resolution of the collision avoidance problem in dynamical environments?. A possible solution may be based on parametrized polyhedra [10] and/or by choosing a particular class of polyhedra, zonotopes [11]. The fundamental idea of such an approach is to concentrate the generators of the shape in a compact form (notable by exploiting the property of symmetry - in the zonotopic case) and to separate them from the parameters affected by the time-variation.

The main contributions of this paper are the following:

i) provide a detailed analysis of evolution of the hyperplane arrangements corresponding to a dynamical multiobstacle environment;

ii) formulate an optimization problem correlated with symmetry properties for time-variant collision avoidance constraints.

The theoretical contributions are exemplified in a prototype control design problem with avoidance-like objectives. Notation: The Minkowski sum of two sets is denoted as $A \oplus B=\{x: x=a+b, a \in A, b \in B\} . \mathcal{C}_{X}(S)$ denotes the complement of the set $S$ over $X \in \mathbb{R}^{d}$. Any polytope (i.e. a bounded polyhedron) has a dual representation in terms of intersection of half-spaces or convex hull of extreme points: $P=\left\{x: s_{i}^{\top} x \leq r_{i}, \forall i\right\}=\left\{x: x=\sum \alpha_{j} v_{j}, \sum \alpha_{j}=\right.$ $\left.1, \alpha_{j} \geq 0, \forall j\right\}$. The set of all sign $N$-tuples is noted $\{-,+\}^{N}:=\left\{\left(b_{1}, \ldots, b_{N}\right): b_{i} \in\{-,+\}, \forall i=1, \ldots, N\right\}$. For $x \in \mathbb{R}^{d}$ we denote $\|x\|_{Q}^{2}=x^{\top} Q x$.

\section{PRELIMINARIES}

Consider a finite collection of time-varying hyperplanes from $\mathbb{R}^{d}$ :

$$
\mathbb{H}(k)=\left\{\mathcal{H}_{i}(k)\right\}_{i \in \mathcal{I}}
$$

where $k$ is the time instant, $\mathcal{H}_{i}(k)=\left\{x \in \mathbb{R}^{d}: s_{i}^{\top} x=\right.$ $\left.r_{i}(k)\right\}$ and $\mathcal{I}=\{1 \ldots N\}$. Each of these hyperplanes divides the space in two disjoint regions:

$$
\mathcal{R}_{i}^{ \pm}(k)=\left\{x \in \mathbb{R}^{d}: \pm s_{i}^{\top} x \leq \pm r_{i}(k)\right\} .
$$

Next, the space can be partitioned into cells using the hyperplane arrangement notion.

Definition 1 (Hyperplane arrangements - [12]): The collection $\mathbb{H}(k)$ partitions the space into a union of 
disjoint cells $\mathcal{A}(\sigma, k)$, characterized by a sign tuple $\sigma \in \Sigma=\{-,+\}^{N}$ :

$$
\mathcal{A}(\sigma, k)=\bigcap_{i \in \mathcal{I}} \mathcal{R}_{i}^{\sigma(i)}(k) .
$$

The hyperplane arrangement of cells covering the entire space is described by the collection of all feasible sign tuples:

$$
\mathcal{A}(\Sigma, k)=\bigcup_{l=1 \ldots 2^{N}} \mathcal{A}\left(\sigma_{l}, k\right),
$$

where $\sigma_{l} \in \Sigma$ is the sign tuple resulting from an intersection of half-spaces.

For the sake of illustration, we depicted in Fig. 1 an arrangement with four hyperplanes in $\mathbb{R}^{2}$, highlighting one of the half-spaces corresponding to $\mathcal{H}_{4}$ and two cells (4).

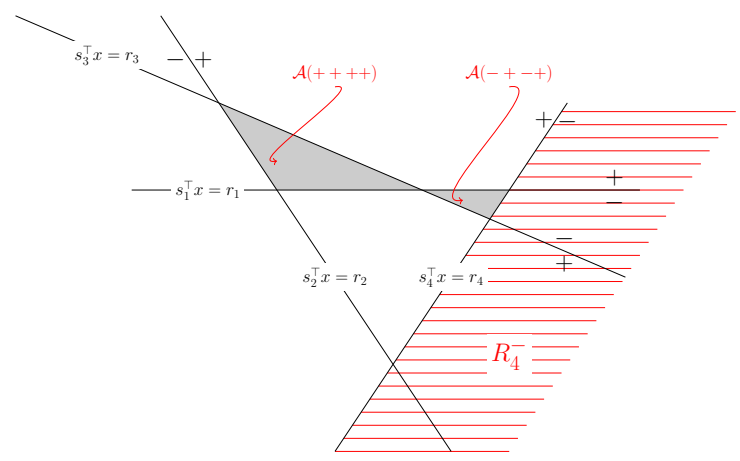

Fig. 1: Hyperplane arrangement $A(\Sigma, k)$.

Remark 1: As can be seen in Fig. 1, there exist sign tuples $\sigma$ for which the cell (3) represents a empty intersection of half-spaces. Therefore, (4) is not necessarily the enumeration of all existing sign combinations, but only of those corresponding to non-empty intersections.

Consider the collection of obstacles (e.g., blue regions in Fig. 2) represented as a union of polyhedral sets

$$
\mathbb{P}_{k}=\bigcup_{j=1}^{N_{o}} P_{j}(k)
$$

with the particularity that each element $P_{j}$ is time-varying and no a priori information is available on the dynamics governing their time-variation.

By employing a MPC (Model Predictive Control) strategy, we aim to obtain a collision free trajectory for an agent, described by the LTI dynamics: $x^{+}=A x+B u$, through a multi-obstacle environment (as the one in Fig. 2).

$$
\begin{aligned}
\min _{\mathbf{u}} \mid & x_{k+N_{p} \mid k}^{T}\left\|_{\mathbf{P}}^{2}+\sum_{l=1}^{N_{p}-1}\right\| x_{k+l \mid k}^{T}\left\|_{\mathbf{Q}}^{2}+\sum_{l=0}^{N_{p}-1}\right\| u_{k+l \mid k}^{T} \|_{\mathbf{R}}^{2} \\
\text { s.t. } & x_{k+l \mid k}=A x_{k+l-1 \mid k}+B u_{k+l-1 \mid k}, \\
& x_{k+l \mid k} \in \mathcal{X}, u_{k+l \mid k} \in \mathcal{U} \\
& x_{k+l \mid k} \in \mathcal{C}_{\mathcal{X}}(\mathbb{P}) .
\end{aligned}
$$

where $N_{p}$ is the prediction horizon, the state and input constraints (6c) are assumed to be convex, containing the origin.
Considering a polytopic representation of the obstacles (5), the non-convex constraints (6d) can be efficiently formulated through a mixed-integer technique [7]. To this end, we gather the collection of support hyperplanes associated to (5), defined as in (1) and we reach the hyperplane arrangement (4). Labeling the feasible cells (3) into interdicted

$$
\Sigma_{\mathbb{P}_{k}}=\left\{\sigma: A(\sigma, k) \cap \mathbb{P}_{k} \neq \emptyset\right\},
$$

or allowed $\Sigma_{X \backslash \mathbb{P}_{k}}=\left\{\sigma: A(\sigma, k) \cap \mathbb{P}_{k}=\emptyset\right\}$, we replace the constraints (6d) with the following mixed-integer formulation:

$$
\begin{aligned}
& s_{i}^{\top} x \leq r_{i}(k)+M\left(1-\alpha_{i}\right), \\
&-s_{i}^{\top} x \leq-r_{i}(k)+M \alpha_{i}, \\
& \sum_{\sigma_{l}(i)=^{\prime}+^{\prime}}\left(1-\alpha_{i}\right)+\sum_{\sigma_{l}(i)==^{\prime}-^{\prime}} \alpha_{i}>0, \forall \sigma_{l} \in \Sigma_{\mathbb{P}_{k}} .
\end{aligned}
$$

where $M$ is a significantly larger constant with respect to the norm of the rest of the variables. Within this big $M$ formulation, $M$ plays the role of a relaxation constant [13].

Since the multi-obstacle environment has a dynamical behavior, the corresponding hyperplane arrangement alters from one instant to another, as it can be noted from the illustration in Fig. 2. However, a re-computation of the arrangement at each sampling instant should be avoided being, on one side, cumbersome from the enumeration point of view but also impractical in a MPC formulation by the topological changes of the feasible domain. For instance, for the simple multi-obstacle environment in Fig. 2, the computation of the feasible cells ${ }^{1}$ (denoted as $\gamma^{*}(N)$ ) requires a considerable amount of time (see Table I), which is in the most of the cases beyond the sampling time associated to the agent dynamics.

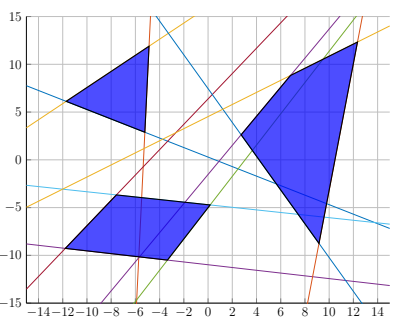

(a) step $k=k_{1}$

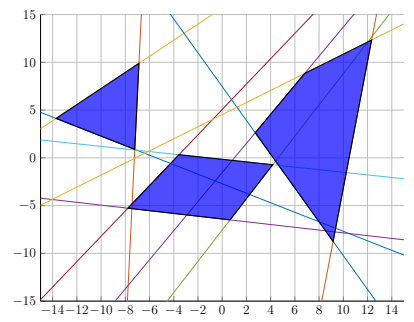

(b) step $k=k_{1}+1$
Fig. 2: Time-varying multi-obstacle environment.

\begin{tabular}{|c|c|c|c|c|c|}
\hline Step & $\# \mathbb{P}$ & $\# \mathbb{H}$ & $\gamma^{*}(N)$ & $t_{\gamma^{*}(N)}[\mathrm{s}]$ & $\# \Sigma_{\mathbb{P}}$ \\
\hline$k=k_{1}$ & 3 & 11 & 49 & 1.04 & 8 \\
\hline$k=k_{1}+1$ & 3 & 11 & 51 & 1.08 & 11 \\
\hline
\end{tabular}

TABLE I: Constructive parameters and computing time of the hyperplanes arrangements given in Fig. 2.

To overcome this issue we point here a technique which introduces an additional analysis step, by evaluating the problem in a lifted space (using a suitable parametrization [10]).

\footnotetext{
${ }^{1}$ We take into account only the cells from the bounded domain $\mathcal{X}$.
} 
This method builds on generic polyhedral sets, but it can be enhanced by using a particular class of polyhedra, namely the zonotopes [11], owing to their symmetry property.

Remark 2: In this paper, in order to concentrate the scope of the work we have examined the case in which the dynamical behavior of the environment consists only in the translation motion of the obstacles.

\section{ZONOTOPIC FRAMEWORK}

Definition 2 (Zonotopes-[11]): A zonotope is a centrally symmetric polytope, which can be described as a Minkowski sum of line segments. In its generator representation a zonotope $\mathcal{Z}(G, c)$ is described by center $c \in \mathbb{R}^{d}$ and generators $G=\left[\begin{array}{lll}g_{1} & \ldots & g_{m}\end{array}\right] \in \mathbb{R}^{d \times m}$ :

$$
\mathcal{Z}(G, c)=\left\{c+\sum_{i=1}^{m} \xi_{i} g_{i}:\|\xi\|_{\infty} \leq 1\right\}
$$

Zonotopes own several properties of practical interest [14]:

i) are closed under Minkowski sum:

$$
\mathcal{Z}\left(G_{1}, c_{1}\right) \oplus \mathcal{Z}\left(G_{2}, c_{2}\right)=\mathcal{Z}\left(\left[\begin{array}{ll}
G_{1} & G_{2}
\end{array}\right], c_{1}+c_{2}\right)
$$

ii) symmetric w.r.t. the center: $-\mathcal{Z}\left(G_{1}, c_{1}\right)=\mathcal{Z}\left(G_{1},-c_{1}\right)$; iii) their volume has an explicit formulation [15]:

$$
\operatorname{Vol}(\mathcal{Z}(G, c))=\sum_{1 \leq j_{1}<j_{2} \ldots j_{d} \leq m}\left|\operatorname{det}\left(G^{j_{1} \ldots j_{d}}\right)\right|,
$$

where $G^{j_{1} \ldots j_{d}}$ denotes the matrix composed from columns of indices $j_{1} \ldots j_{d}$ taken from $G$.

Having the generator form of a zonotope, the half-space representation can be constructed as follows [16]: to each sequence of $d-1$ generators $1 \leq j_{1}<j_{2} \ldots j_{d-1} \leq m$ corresponds the pair $\left(h_{i}, k_{i}\right) \in \mathbb{R}^{d} \times \mathbb{R}$, where:

$$
s_{i} \perp g_{j_{l}}, \forall j_{l} \in\left\{j_{1} \ldots j_{d-1}\right\}, \quad r_{i}=\sum_{j_{l} \notin\left\{j_{1} \ldots j_{d-1}\right\}}\left|s_{i}^{\top} g_{j_{l}}\right|
$$

Adding the center from generator representation, a zonotope is formulated as:

$$
\mathcal{Z}(G, c)=\bigcap_{1 \leq j_{1}<\ldots j_{d-1} \leq m}\left\{x \in \mathbb{R}^{d}:\left|s_{i}^{\top}(x-c)\right| \leq r_{i}\right\} .
$$

The initial collection of obstacles (5) becomes:

$$
\mathbb{Z}_{k}=\bigcup_{j=1}^{N_{o}} \mathcal{Z}_{j}(k)
$$

where $\mathcal{Z}_{j}(k)$ represents the result of an over-approximation procedure. There exists in the literature a variety of algorithms providing over-approximations [16] and many of them consider pre-defined families of zonotopes (e.g., by fixing the directions of the generators).

Considering Def. 2 and (9) we refer to a family of zonotopes parametrized after their centers $c_{\ell} \in \mathbb{R}^{d}$ and scaling factors $\Delta_{\ell} \in \mathbb{R}^{m \times m}$ applied to a common generator "seed" (an a priori given matrix $G \in \mathbb{R}^{d \times m}$ ):

$$
\mathcal{Z}\left(G \Delta_{j}, c_{j}\right), \quad j=1 \ldots N_{o}
$$

$\Delta_{\ell}$ is a diagonal matrix whose diagonal elements are equal $/$ distinct $^{2}$. The k-th diagonal element is noted as $\delta_{j_{k}}$.

Having a common generator seed has numerous advantages in what regards the over-approximation procedure and, moreover, regarding the complexity of the control problem.

Therefore, the overall goal is to provide adequate overapproximations (15) for the multi-obstacle environment (5). That is, seek a (inherently symmetric) zonotope $\mathcal{Z}\left(G \Delta_{j}, c_{j}\right)$ enclosing the known (usually non-symmetric) polytope $P_{j}$ such that a specific measure parametrized after $c_{j}, \Delta_{j}$ is minimized:

$$
\begin{aligned}
\left(\Delta_{j}, c_{j}\right)^{*}= & \arg \min _{\Delta_{j}, c_{j}} \mathcal{C}\left(\Delta_{j}, c_{j}\right) \\
& \text { s.t. } P_{j} \subseteq \mathcal{Z}\left(G \Delta_{j}, c_{j}\right) .
\end{aligned}
$$

The inclusion (16b) can be readily expressed using the support functions [17] in combination with the definition of a zonotope:

$$
\left|s_{i}^{\top}\left(v_{k}-c_{j}\right)\right| \leq r_{i}\left(\Delta_{j}\right), \forall k .
$$

where $s_{i}$ and $r_{i}\left(\Delta_{j}\right)$ are given by the half-space representation of the i-th zonotope from (15), replacing $g_{k}$ with $g_{k} \cdot \delta_{j_{k}}$ in (12). Several measures can be considered [18] with different performances, in this paper we opted to use the measure which provides the most accurate (tightest) approximation, specifically the one given by the volume (11):

$$
\mathcal{C}\left(\Delta_{j}, c_{j}\right)=\sum_{1 \leq k_{1}<\cdots<k_{d} \leq m}\left|\operatorname{det}\left(G^{k_{1} \ldots k_{d}}\right)\right| \cdot \prod_{k \in\left\{k_{1}, \ldots, k_{d}\right\}} \delta_{j_{k}} .
$$

One of the most relevant benefit of using a common generator seed is that it provides some complexity bounds of the representation of the multi-obstacle environment [18].

\section{PARAMETRIZED HYPERPLANE ARRANGEMENTS}

As stated in Section I, the collection (1) can evolve due to the dynamical behavior of the environment, or in other words whenever the obstacles change their position in time. For this reason and in order to avoid a burdensome re-computation, a pre-analysis is necessary.

Let us consider a parameter $p \in \mathbb{R}^{n_{p}}$ and a linear dependence of the polyhedral set description:

$$
\mathcal{H}_{i}(p)=\left\{x \in \mathbb{R}^{d}: s_{i}^{\top} x=r_{i}-\left(s_{i}^{p}\right)^{\top} p\right\},
$$

Remark 3: Specifically, in (19) we separate the timevarying component of the hyperplanes in (1): $r_{i}(k)=r_{i}-$ $\left(s_{i}^{p}\right)^{\top} p(k)$. For compactness, $k$ was omitted in (19).

Hence, we have a collection of hyperplanes in the lifted space $\mathbb{H}^{*}=\left\{\mathcal{H}_{i}^{*}\right\} \in \mathbb{R}^{d+n_{p}}$ with:

$$
\mathcal{H}_{i}^{*}=\left\{x \in \mathbb{R}^{d+n_{p}}:\left[\begin{array}{ll}
s_{i}^{\top} & \left(s_{i}^{p}\right)^{\top}
\end{array}\right]\left[\begin{array}{l}
x \\
p
\end{array}\right]=r_{i}\right\},
$$

The arrangement $\mathcal{A}\left(\mathbb{H}^{*}\right)$ is composed of cells $\mathcal{A}\left(\sigma^{*}\right)$. The parametrization (19) leads to a dependence of the domain of existence of each cell $\mathcal{A}\left(\sigma^{*}\right)$ w.r.t. the parameter $p$.

\footnotetext{
${ }^{2}$ If not explicitly stated otherwise, we consider the later case since the former is a simplification of the later.
} 
Therefore, we need to project on the parameter space in order to describe the range of variation of the respective configuration. Conversely, by cutting the polyhedron (20) at a particular value $p$ leads to a parameterized form of the arrangement $\mathcal{A}(\sigma, p)$.

Recalling the halfspace representation of a zonotope (13), we note that the hyperplanes defining a zonotope are strongly dependent on the value of the zonotope center. Therefore, these hyperplanes can be written under the following form:

$$
\mathcal{H}_{i}(c)=\left\{x \in \mathbb{R}^{d}: s_{i}^{\top} x=r_{i}+s_{i}^{\top} c\right\},
$$

Consequently, the center $c$ is a suitable choice as the parameter $p$ in (19) or as one of its component. Noteworthy, in the context of the translation motion, $c$ is the only time-varying characteristic of a zonotope.

Next, we rewrite (20) for the case $p \leftarrow c$ :

$$
\mathcal{H}_{i}^{*}=\left\{\left[\begin{array}{l}
x \\
c
\end{array}\right] \in \mathbb{R}^{2 d}:\left[\begin{array}{ll}
s_{i} & -s_{i}
\end{array}\right]\left[\begin{array}{l}
x \\
c
\end{array}\right]=r_{i}\right\}
$$

The arrangement $\mathcal{A}\left(\mathbb{H}^{*}\right)$ with $\mathbb{H}^{*}(c)=\left\{\mathcal{H}_{i}^{*}(c)\right\}$ is composed of feasible cells $\mathcal{A}\left(\sigma^{*}\right), \sigma^{*} \in \Sigma^{*}$. An interesting feature of this approach is that it allows to compute the domain of existence of each cell $\mathcal{A}\left(\sigma^{*}\right)$ w.r.t. the center position by projecting on the parameter (center) space:

$$
\operatorname{dom}\left\{\mathcal{A}\left(\sigma^{*}\right)\right\}=\left\{c: \exists x \quad \text { s.t. }\left[\begin{array}{l}
x \\
c
\end{array}\right] \in \mathcal{A}\left(\sigma^{*}\right)\right\}
$$

All these validity domains can be computed offline and represent polyhedral domains in $\mathbb{R}^{d}$.

The above reasoning can lay an instrumental role in the next multi-agent scenario. Let us consider two zonotopes: $Z_{1}=\mathcal{Z}\left(G_{1}, c_{1}\right), Z_{2}=\mathcal{Z}\left(G_{2}, c_{2}\right)$ with their compact halfspace representations:

$Z_{1}=\left\{x: H_{1}\left(x-c_{1}\right) \leq w_{1}\right\}, \quad Z_{2}=\left\{x: H_{2}\left(x-c_{2}\right) \leq w_{2}\right\}$

A cell $\mathcal{A}\left(\sigma^{\prime}\right)$ from the hyperplane arrangement induced by these two zonotopes is given by:

$$
\begin{aligned}
\mathcal{A}\left(\sigma^{\prime}\right)=\left\{x \in \mathbb{R}^{n}: \quad\right. & H_{1}^{\sigma^{i}}\left(x-c_{1}\right) \leq w_{1}^{\sigma^{i}}, \\
& \left.H_{2}^{\sigma^{i}}\left(x-c_{2}\right) \leq w_{2}^{\sigma^{i}}\right\}
\end{aligned}
$$

where $\sigma^{\prime}=\left[\begin{array}{ll}\sigma^{1} & \sigma^{2}\end{array}\right]$ with $\sigma^{i}$ sign tuple w.r.t. $Z_{i}, i \in\{1,2\}$.

Note that the important aspect at the above described multi-agent scenario is not the absolute position of the centers, but their relative position. Thus, we can assume, without loss of generality, that the obstacle $Z_{1}$ is fixed and $Z_{2}$ is a moving obstacle. Hence we can construct the correspondent collection of hyperplanes $\hat{\mathbb{H}}(c)=\{\hat{\mathcal{H}}\}$ :

$$
\begin{aligned}
\hat{\mathcal{H}}= & \left\{\left[\begin{array}{c}
x \\
c_{2}
\end{array}\right] \in \mathbb{R}^{2 n}:\right. \\
& {\left.\left[\begin{array}{cc}
H_{1}^{\sigma^{i}} & 0 \\
H_{2}^{\sigma^{j}} & -H_{2}^{\sigma^{j}}
\end{array}\right]\left[\begin{array}{c}
x \\
c_{2}
\end{array}\right] \leq\left[\begin{array}{c}
w_{1}^{\sigma^{i}}+H_{1}^{\sigma^{i}} c_{1} \\
w_{2}^{\sigma^{j}}
\end{array}\right]\right\} }
\end{aligned}
$$

Similarly to (23), we compute the domain of existence of each cell $\mathcal{A}\left(\sigma^{\prime}\right)$ in the parameter $c_{2}$ space (validity domain)[10]:

$$
\operatorname{dom}\left(\mathcal{A}\left(\sigma^{\prime}\right)\right)=\operatorname{proj}_{c_{2}} \mathcal{A}\left(\sigma^{*}\right)
$$

\section{Illustrative example}

Consider the hyperplane arrangement induced by the following two zonotopes. Using this method for a simple case ( $d=2$, "rectangular" zonotopes Fig. 3a) we obtain that only 81 tuples are feasible ( $\exists c_{2}$ such that the tuple is feasible) from $2^{8}=256$ possible ones. Moreover, the symmetry of the zonotopes brings a benefit with respect to the number of valid domains Fig. 3.

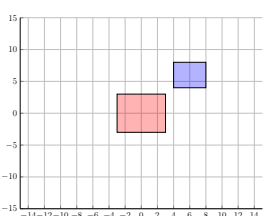

(a) Zonotopes (24)

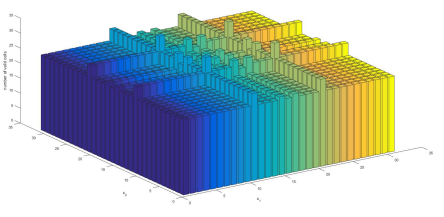

(b) Number of validity domains
Fig. 3: Illustrative example of the parametrization of the hyperplane arrangements. The left figure presents one particular position of the obstacles for a given value of the parameters. On the right, the number of regions describing the complement of the obstacles as a function of the interdistance between their centers.

Remark 4: We can further extend the method for a general case consisting of $N \geq 3$ dynamical obstacles. Thus, considering the relative position of the centers with respect to a 'fixed' one (e.g., the $N$-th obstacle), we obtain a collection of hyperplanes as in (22), except that the lifted space is augmented towards $\mathbb{R}^{d+(N-1) d}$. In other words, $c \mapsto\left[\begin{array}{llll}c_{1}^{\top} & c_{2}^{\top} & \ldots & c_{N-1}^{\top}\end{array}\right]^{\top}$. Certainly, this may lead to a relatively high complexity, but it is a desirable aspect compared to the re-computation of the arrangement at each sampling instant.

\section{Collision AVOIDANCE With MOVING OBSTACLES}

In order to illustrate the valuable insight of the hyperplane parametrization in the control problem we consider a case study which can be interpreted as a surveillance mission with respect to two intruders. More precisely, an agent has to monitor these two intruders and but also to remain outside their region of visibility. The agent is described by the LTI dynamics (6b) in $\mathbb{R}^{d}, d=3$, with the following parameters:

$$
A=\left[\begin{array}{cc}
O_{d} & I_{d} \\
O_{d} & -\frac{\mu}{M} I_{d}
\end{array}\right], \quad B=\left[\begin{array}{c}
O_{d} \\
M_{a}^{-1} I_{d}
\end{array}\right],
$$

where $\mu=3$ and $M_{a}=60$. The agent's state is composed from position and velocity components

$$
x=\left[\begin{array}{llllll}
p_{x} & p_{y} & p_{z} & v_{x} & v_{y} & v_{z}
\end{array}\right]^{\top},
$$

whereas the input is given by the acceleration

$$
u=\left[\begin{array}{lll}
a_{x} & a_{y} & a_{z}
\end{array}\right]^{\top} .
$$

Both state and input are constrained: $\mathcal{X}=\left\{x:-15 \leq x_{i} \leq\right.$ $\left.15, \forall i=1 \ldots 2 d, x_{2 d-1}=p_{z} \geq 0\right\}$ and $\mathcal{U}=\{u:-3 \leq$ $\left.u_{i} \leq 3, \forall i=1 \ldots 2 d\right\}$. For the monitoring ability, the agent 
is equipped with downwards facing visual sensors [19], able to cover a conical field of view. Hence, the agent is able to survey (in a xy-plane) an ellipsoidal region centered in $\left[\begin{array}{ll}p_{x} & p_{y}\end{array}\right]$, whose dimension depends on the value of $p_{z}$ and on the characteristics of the visual sensor (hereinafter, we consider the field of view angle $\phi$ ).

The two intruders (and their visibility zones) are modeled using two convex bodies with uncorrelated motions, whose trajectories are depicted in Fig. 4. Since the motion is within the xy-plane, the resulting interdicted regions can be defined as zonotopes by taking a common generator matrix $^{3} G=\left[\begin{array}{ll}1 & 0 \\ 0 & 1\end{array}\right]$ and two different scaling factors $\Delta_{1}=\operatorname{diag}\left(\left[\begin{array}{ll}2.5 & 3\end{array}\right]\right)$ and $\Delta_{2}=\operatorname{diag}\left(\left[\begin{array}{ll}1 & 0.5\end{array}\right]\right)$, while the centers, $c_{1}(k)$ and $c_{2}(k)$ respectively, follow the trajectories depicted in Fig. 4. For further use we denote:

$$
\mathbb{P}_{k}=\mathcal{Z}\left(G \Delta_{1}, c_{1}(k)\right) \cup \mathcal{Z}\left(G \Delta_{2}, c_{2}(k)\right)
$$

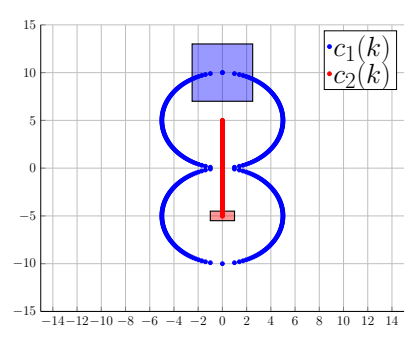

(a) $\mathrm{X}-\mathrm{Y}$

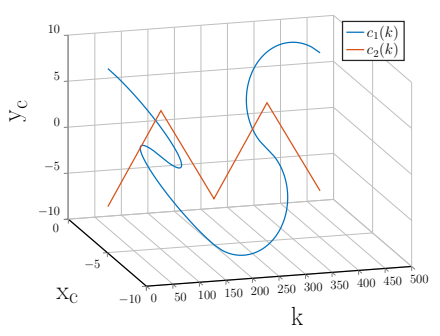

(b) X-Y-t
Fig. 4: Trajectories of the two intruders.

The scenario depicted in Fig. 4 will be considered for the test cases such that the motion of the intruders is cyclical and restricted to the xy-plane. Therefore, we deal with the surveillance mission by partially decoupling the $\mathrm{xy}$-dimensions and z-dimension respectively. We delineate in Fig. 5 the control scheme corresponding to the proposed method. Firstly, we have two blocks which provide information about the dynamical multi-obstacle environment and set the reference points. Based on these we set as objective of the control design the tracking of the midpoint between two moving bodies (obstacles, detection zones, etc.):

$$
\bar{\chi}_{\mathrm{ref} \mid k}=\frac{1}{2}\left(c_{1}(k)+c_{2}(k)\right) \text {. }
$$

The $\mathrm{MPC}_{\mathrm{xy}}$ consists in the resolution of a constrained optimization (30). Hence, if the intersection of the agent coordinates in xy-plane with the intruders' neighborhood (pre-defined visibility zones) cannot be avoided, it should be minimized. From the MPC's point of view this objective can be translated in hard avoidance constraints. But, in order to avoid the infeasibility of such constraints, we penalize the entry into these zones in the objective function associated

\footnotetext{
${ }^{3}$ For the sake of clarity, we considered two "boxes", but the reasoning and simulation are generic and apply also for more complex shapes of the obstacle, in as much as they are (approximated by) zonotopic sets.
}

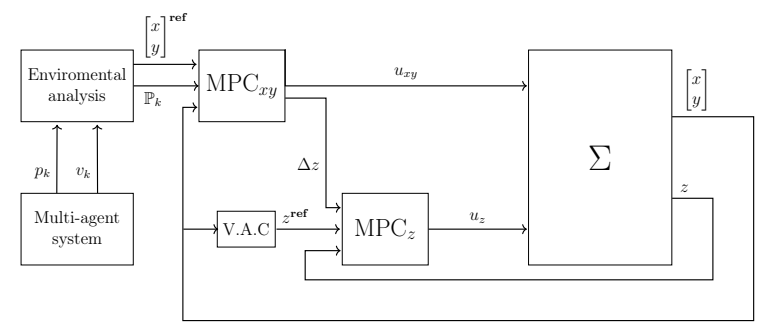

Fig. 5: The control scheme.

to the MPC problem, as in (31c). Any violation of the constraints in the $x y$ coordinates should be made at a considerably higher height. By consequence, whenever the $x y$ anti-collision constraints are violated, they are translated in an increase of the reference in the $z$-coordinated which represents an "escape" dimension. Practically, this penalty will be further employed in the MPC problem corresponding to the third dimension.

$$
\begin{aligned}
& \min _{\mathbf{u}^{x y}, \epsilon}\left(\left\|\chi_{k+N_{p} \mid k}-\bar{\chi}_{\mathrm{ref} \mid k}\right\|_{\mathbf{P}}^{2}\right.+\sum_{l=1}^{N_{p}-1}\left\|\chi_{k+l \mid k}-\bar{\chi}_{\mathrm{ref} \mid k}\right\|_{\mathbf{Q}}^{2}+ \\
&\left.+\sum_{l=0}^{N_{p}-1}\left\|\Delta u_{k+l \mid k}^{x y}\right\|_{\mathbf{R}}^{2}+\epsilon M\right) \\
& \text { s.t. } \quad \chi_{k+l \mid k}=A_{x y} \chi_{k+l-1 \mid k}+B_{x y} u_{k+l-1 \mid k}^{x y}, \\
& \chi_{k+l \mid k} \in \mathcal{X}^{x y}, u_{k+l \mid k}^{x y} \in \mathcal{U}^{x y}, \\
& \chi_{k+l \mid k}+\epsilon \notin \mathbb{P}_{k},
\end{aligned}
$$

Remark 5: In (31) the state vector $\chi$ is composed from the position and velocity corresponding to the $\mathrm{x}-\mathrm{y}$-dimensions, and the matrices $A_{x y}, B_{x y}$ are their associated values from (27). This holds likewise for input and for sets $\mathcal{X}^{x y}$, $\mathcal{U}^{x y}$, which are projections of the initial sets on the xysubspace. Furthermore, $M$ represents a sufficiently large constant, which includes the conversion of units between $x y$ coordinates and $z$-coordinates.

The existence of the common "seed" $G$ in (28) allows us to rewrite the control problem (30) obtaining a parametrization with respect to the positions of the centers. Further using a similar procedure like in the case of (6d), we rewrite (31c) as mixed-integer constraints like in (8) and the resulting MI problem can be solved using, e.g., CPLEX [20].

Regarding the block $\mathrm{MPC}_{\mathrm{z}}$, the control strategy relies on resolution of (32).

$$
\begin{array}{ll}
\min _{\mathbf{u}^{z}} & \left(\sum_{l=1}^{N_{p}-1}\left\|z_{k+l \mid k}-\bar{z}_{\mathrm{ref} \mid k}\right\|_{\mathbf{Q}_{\mathbf{z}}}^{2}\right) \\
\text { s.t. } & z_{k+l \mid k}=A_{z} x_{k+l-1 \mid k}+B_{z} u_{k+l-1 \mid k}^{z}, \\
& z_{k+l \mid k} \in \mathcal{Z}, \tilde{u}_{k+l \mid k} \in \mathcal{U}_{z}, \\
& z_{k+l+2} \geq \bar{z}_{\mathrm{ref} \mid k} .
\end{array}
$$

where $A_{z}, B_{z}$ are selected from the extended system (27). The reference $\bar{z}_{\text {ref } \mid k}$ has the following components: $p_{z \mid k}^{\text {ref }}$ which is the sum between the reference given by the visual 
area coverage (dependent on the xy-position) $z_{k}^{\text {ref }}$ and the value of the penalty $\epsilon$ resulted from $\mathrm{MPC}_{\mathrm{xy}}$; and $v_{z \mid k}^{\text {ref }}$, which is set at 0 . The constraint (32d) ensures that the field of view of the agent includes the zone of interest, even though not at the optimal quality.

The computation of $z_{k}^{\text {ref }}$ (the block V.A.C. $=$ visual area coverage) is based on finding the minimal ellipsoid centered in $p_{x y}=\left[\begin{array}{ll}p_{x} & p_{y}\end{array}\right]$ which is able to (visual) cover (at optimal quality) all possible position of the intruders (the zone of interest). To this end, we need to solve the following optimization problem [21] at each sampling instant:

$$
\begin{aligned}
& z_{k}^{\text {ref }}=\min _{p_{z}} p_{z} \\
& \text { s.t. } \frac{1}{p_{z} \tan \phi}\left\|\nu_{i}-p_{x y}\right\| \leq 1, \forall i=1 \ldots n_{v},
\end{aligned}
$$

where $\nu_{i}$ represents the vertices of the convex hull of the surveyed zone, and $\phi$ is the angle of the conical field of view.

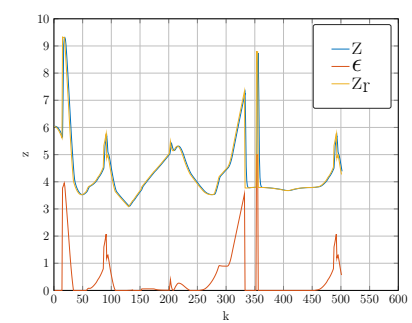

(a) $p_{z}, \epsilon$

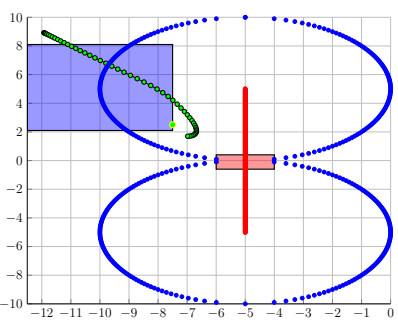

(b) $\mathrm{X}-\mathrm{t}$
Fig. 6: Simulation results for $N_{p}=10 P=10^{4} I_{2 d}, Q=$ $10^{6} I_{2 d}, R=I_{d}$ and $Q_{z}=10^{5} I_{2 d}$.

In Fig. 6a the time-variation of the value of $\epsilon$ and of the value $p_{z}$ are shown. Note that, it can be visible in the $\mathrm{z}$ coordinate when the anti-collision constraints (30) do not hold, while ensuring an optimal monitoring of the zone of interest.

\section{CONCLUSIONS}

We have tackled the collision avoidance problem within a dynamical multi-obstacle environment by employing an optimization-based control strategy. The main contribution is the use of parametrized hyperplane arrangements and zonotopic sets in order to obtain a compact representation of the resulting time-variant non-convex feasible domain in terms of mixed-integer constraints. Lastly, we apply the result over a prototype surveillance problem. The challenges with respect to the use of a parametrized version of the obstacles and the related hyperplane arrangements is the computation, storage and location within the validity domains. Obviously, in the case of dynamical environments with multiple obstacles, a prior stage of identification of the closest obstacles is to be considered in order to reduce the exponential complexity of the parameter space and of the subsequent validity domains.

\section{ACKNOWLEDGMENTS}

The research of Daniel Ioan is financially supported by the Ministry of the Armed Forces - Defence Procurement
Agency (DGA) - no.2017352. In particular, the authors acknowledge Jacques Blanc-Talon, expert DGA, for the fruitful discussions.

The research of Florin Stoican is supported by the Politehnica University of Bucharest, through the internal research grant GNaC 2918 ARUT, contract no. 4/15.10.2018

\section{REFERENCES}

[1] F. Janecek, M. Klauco, M. Kaluz, and M. Kvasnica, "OPTIPLAN: A Matlab Toolbox for Model Predictive Control with Obstacle Avoidance," IFAC-PapersOnLine, vol. 50, no. 1, pp. 531-536, Jul. 2017.

[2] S. Karaman and E. Frazzoli, "Sampling-based algorithms for optimal motion planning," The International Journal of Robotics Research, vol. 30, no. 7, pp. 846-894, Jun. 2011.

[3] A. Richards and J. P. How, "Aircraft trajectory planning with collision avoidance using mixed integer linear programming," in American Control Conference, 2002. Proceedings of the 2002, vol. 3. IEEE, 2002, pp. 1936-1941.

[4] F. Stoican, I. Prodan, and S. Olaru, "Hyperplane arrangements in mixed-integer programming techniques. Collision avoidance application with zonotopic sets," in 2013 European Control Conference (ECC), Jul. 2013, pp. 3155-3160.

[5] Y.-b. Chen, G.-c. Luo, Y.-s. Mei, J.-q. Yu, and X.-1. Su, "UAV path planning using artificial potential field method updated by optimal control theory," International Journal of Systems Science, vol. 47, no. 6, pp. 1407-1420, Apr. 2016.

[6] R. Olfati-Saber and R. M. Murray, "Distributed cooperative control of multiple vehicle formations using structural potential functions," in IFAC world congress, vol. 15, no. 1, 2002, pp. 242-248.

[7] I. Prodan, F. Stoican, S. Olaru, and S.-I. Niculescu, "Mixed-Integer Representations," in Mixed-Integer Representations in Control Design, ser. SpringerBriefs,Engineering. Springer, Cham, 2016.

[8] I. Ballesteros-Tolosana, S. Olaru, P. Rodriguez-Ayerbe, G. Pita-Gil, and R. Deborne, "Collision-free trajectory planning for overtaking on highways," in 2017 IEEE 56th Annual Conference on Decision and Control (CDC), Dec. 2017, pp. 2551-2556.

[9] R. Hult, M. Zanon, S. Gros, and P. Falcone, "An miqp-based heuristic for optimal coordination of vehicles at intersections," in Proc. 57th Conf. Decis. Control (CDC).

[10] S. B. Olaru and D. Dumur, "A parameterized polyhedra approach for explicit constrained predictive control," in Decision and Control, 2004. CDC. 43rd IEEE Conference on, vol. 2. IEEE, 2004, pp. 1580-1585.

[11] W. Kuhn, "Rigorously computed orbits of dynamical systems without the wrapping effect," Computing, vol. 61, no. 1, pp. 47-67, Mar. 1998.

[12] G. M. Ziegler, Lectures on polytopes. Springer Science \& Business Media, 2012, vol. 152.

[13] J. P. Vielma and G. L. Nemhauser, "Modeling disjunctive constraints with a logarithmic number of binary variables and constraints," Mathematical Programming, vol. 128, no. 1-2, pp. 49-72, 2011.

[14] K. Fukuda, "From the zonotope construction to the Minkowski addition of convex polytopes," Journal of Symbolic Computation, vol. 38, pp. 1261-1272, Oct. 2004.

[15] E. Gover and N. Krikorian, "Determinants and the volumes of parallelotopes and zonotopes," Linear Algebra and its Applications, vol. 433, no. 1, pp. 28-40, Jul. 2010.

[16] M. Althoff, O. Stursberg, and M. Buss, "Computing reachable sets of hybrid systems using a combination of zonotopes and polytopes," Nonlinear analysis: hybrid systems, vol. 4, no. 2, pp. 233-249, 2010.

[17] I. Kolmanovsky and E. G. Gilbert, "Theory and computation of disturbance invariant sets for discrete-time linear systems," Mathematical problems in engineering, vol. 4, no. 4, pp. 317-367, 1998.

[18] D. Ioan, I. Prodan, F. Stoican, S. Olaru, and S.-I. Niculescu, "Complexity bounds for obstacle avoidance within a zonotopic framework," L2S, CNRS, Supelec, Universit Paris Sud. 2019, Tech. Rep., 012019.

[19] S. Papatheodorou, A. Tzes, and Y. Stergiopoulos, "Collaborative visual area coverage," Robotics and Autonomous Systems, vol. 92, pp. 126138, 2017.

[20] I. I. CPLEX, "V12. 1: Users manual for cplex," International Business Machines Corporation, vol. 46, no. 53, p. 157, 2009.

[21] S. Boyd and L. Vandenberghe, Convex optimization. Cambridge university press, 2004. 